\title{
Using Mobile Touch Devices to Provide Flexible Classroom Assessment Techniques
}

\author{
Séamus C. McLoone, Department of Electronic Engineering, Maynooth University, \\ Maynooth, Ireland
}

Rudi Villing, Department of Electronic Engineering, Maynooth University, Maynooth, Ireland

Simon O'Keeffe, Department of Electronic Engineering, Maynooth University, Maynooth, Ireland

\begin{abstract}
Classroom assessment techniques (CATs) are ungraded activities in a classroom setting that provide feedback to the teacher and to the students themselves, on the current state of student learning and understanding, which can subsequently drive corrective actions where necessary. Student response systems (SRSs) provide a technological solution for CATs whereby students can respond anonymously and instructors can provide instant feedback. However, existing systems have tended to suffer from constrained input, limiting the quality of the student responses. In particular, existing SRSs typically only employ well known form based input metaphors such as the multiple-choice selection and text-box input. These input types are not well suited to responses that require significant graphic or symbolic elements such as equations, circuit diagrams, and other drawings. These SRSs also have logistical issues in relation to portability and ownership of the equipment. In this paper, the authors present an SRS designed from the ground up to support CATs with freeform input to fulfil the needs of the science, technology, engineering and mathematics (STEM) classroom, though the solution is applicable to any learning environment in which freeform input is valuable. To mitigate logistical issues, the solution employs touch based Android tablets and smart phones commonly owned by students and a freely downloadable student app. This paper details the design of teacher and student interaction, including instructor preparation prior to class. The authors also examine some of the issues surrounding freeform graphic and symbolic input on a range of device form factors and the particular solutions that they found effective. A summary of their ongoing evaluation of this system is also outlined within.
\end{abstract}

Keywords: $\quad$ Audience Response Systems, Classroom Assessment Techniques (CATS), Smart-Device Based Response Systems, Student Response Systems, Technology in the Classroom

DOI: 10.4018/IJMHCI.2015100101 


\section{INTRODUCTION}

As teachers, we are all well aware that the final examination occurs too late to be of any use in addressing the learning needs that our students may have had during the taught module or programme. Classroom Assessment Techniques (CATs) (Angelo \& Cross, 1993) offer a solution in this regards, as these formative and ungraded activities are designed to provide almost immediate feedback to the teacher and, indeed, to the students themselves about what the students are learning and what deficiencies may exist in their knowledge and understanding of the current material. Obtaining this information allows the teacher to take justin-time corrective action to address any such deficiencies and, thus, improve and enhance the student learning experience. To date, the most comprehensive overview of CATs has been carried out by Angelo and Cross (1993), who present fifty different such techniques. Traditional examples include the one-minute paper (a frequently employed CAT that requires students to answer two questions, namely what was the most important thing learned and what remains unanswered), the muddiest point (students are required to answer what the muddiest point in a lecture or a section of notes, for example, was), the classroom opinion poll and the student-generated test questions.

The CATs are typically conducted using paper and pen and often carried out in one lecture, with the information processed and reviewed after the lecture and subsequent action carried out in the following lecture. The advent of student response systems (SRSs) (Fies \& Marshall, 2006; MacArthur \& Jones, 2008; Kay \& LeSage, 2009; Blasco-Arcas et al., 2013) provided a technological solution for the implementation of a selection of the CATs (Briggs \& Keyek-Franssen, 2010) that significantly simplified the data gathering and reviewing process. In addition, students could truly respond anonymously (as the issue of recognising one's handwriting was no longer a possibility) and in the case of some of the CATs (such as the classroom opinion poll), feedback could be obtained practically instantly.

Student response systems exist in many different guises, including audience response systems (Miller etal., 2003), classroom response systems (Roschelle, 2004), voting machines (Reay et al., 2005; Simpson \& Oliver, 2007), clicker assessment and feedback technology (CAF) (Han \& Finkelstein, 2013), classroom communication systems (Boyle \& Nicol, 2003), and clickers (Barber \& Njus, 2007; Lantz, 2010). All such systems combine software and hardware to allow lecturers to pose questions to their students and to obtain almost instant feedback in real-time, i.e. there and then within the classroom. The typical setup consists of a handheld transmitter unit for use by the students, a receiver unit that collates the student responses and suitable software to present the responses in a user-friendly form. An example of such a response system is shown in Figure 1 (Barber \& Njus, 2007).

The numerous educational benefits of using student response systems within the classroom are well documented in the literature. Along with providing an easier implementation for CATs, they can also improve active learning, improve student motivation, increase student attendance, improve student interaction within the classroom, increase student preparation for classes and improve student satisfaction with the learning environment (Faust \& Pauslon, 1998; Sarason \& Banbury, 2004; Skiba, 2006; Caldwell, 2007; Auras \& Bix, 2007; Moredich \& Moore, 2007; Blood \& Neel, 2008; Hoekstra, 2008; Bruff, 2009; Heaslip et al., 2014). They also offer anonymity which increases student participation when using such systems to respond to questions posed by lecturers (Graham et al., 2007).

However, existing systems suffer from constrained input which typically involves a multiple choice selection or a text-box input and thus limits the individual feedback that could be obtained. These input types do not cater for graphic or symbolic elements such as mathematical equations, circuit diagrams, flow charts, etc., all of which are particularly 
13 more pages are available in the full version of this document, which may be purchased using the "Add to Cart" button on the product's webpage:

www.igi-global.com/article/using-mobile-touch-devices-toprovide-flexible-classroom-assessmenttechniques $/ 132648$ ?camid $=4 \mathrm{v} 1$

This title is available in InfoSci-Journals, InfoSci-Journal

Disciplines Communications and Social Science, InfoSciSelect, InfoSci-Select, InfoSci-Technology Adoption, Ethics, and Human Computer Interaction eJournal Collection, InfoSci-Networking, Mobile Applications, and Web Technologies eJournal Collection, InfoSci-Journal Disciplines Computer Science, Security, and Information Technology, InfoSci-Journal Disciplines Engineering, Natural, and Physical Science. Recommend this product to your librarian: www.igi-global.com/e-resources/libraryrecommendation/?id=2

\section{Related Content}

Innovative Approaches in Pair Programming to Enhance the Quality of Software Development

Naresh E. and Vijaya Kumar B.P. (2018). International Journal of Information Communication Technologies and Human Development (pp. 42-53). www.igi-global.com/article/innovative-approaches-in-pair-programming-toenhance-the-quality-of-software-development/206562?camid=4v1a

Computer Access for Motor-Impaired Users

Shari Trewin and Simeon Keates (2006). Encyclopedia of Human Computer Interaction (pp. 92-99).

www.igi-global.com/chapter/computer-access-motor-impairedusers $/ 13106$ ?camid $=4 \mathrm{v} 1 \mathrm{a}$ 
Online Learning Readiness: Its Relations to College Students' Changes over Time, and Willingness to Enroll in Future Courses

Min-Ling Hung (2016). International Journal of Technology and Human Interaction (pp. 51-62).

www.igi-global.com/article/online-learning-readiness/144319?camid=4v1a

Is Information Ethics Culture-Relative?

Philip Brey (2007). International Journal of Technology and Human Interaction (pp. 12-24).

www.igi-global.com/article/information-ethics-culturerelative/2904?camid $=4 \mathrm{v} 1 \mathrm{a}$ 